\title{
Characteristics of tongue and pharyngeal pressure in patients with neuromuscular diseases
}

This article was published in the following Dove Press journal:

Degenerative Neurological and Neuromuscular Disease

30 May 2017

Number of times this article has been viewed

\section{George Umemoto' \\ Hirokazu Furuya ${ }^{2}$ \\ Yoshio Tsuboi ${ }^{3}$ \\ Shinsuke Fujioka ${ }^{3}$ \\ Hajime Arahata ${ }^{4}$ \\ Miwa Sugahara ${ }^{4}$ \\ Mitsuaki Sakai ${ }^{5}$}

'Department of Oral and Maxillofacial Surgery, Faculty of Medicine, Fukuoka University, Fukuoka, ${ }^{2}$ Department of Neurology, Faculty of Medicine, Kochi University, Kochi, ${ }^{3}$ Department of Neurology, Faculty of Medicine, Fukuoka University, ${ }^{4}$ Department of Neurology, ${ }^{5}$ Department of Rehabilitation, Neuro-Muscular Center, National Omuta Hospital, Fukuoka, Japan
Correspondence: George Umemoto Department of Oral and Maxillofacial Surgery, Faculty of Medicine, Fukuoka University, 7-45-I Nanakuma, Jonan-ku, Fukuoka 8I4-0I80, Japan

$\mathrm{Tel}+8 \mid 9280$ I I0I I

Fax +8I 928011044

Email george@adm.fukuoka-u.ac.jp
Background: Tongue and pharyngeal pressure is an essential factor associated with the swallowing function; however, little is known about the difference in tongue and pharyngeal pressure between neuromuscular diseases. This study aimed to characterize tongue and pharyngeal pressure in myotonic dystrophy type 1 (DM1), Duchenne muscular dystrophy (DMD), and amyotrophic lateral sclerosis (ALS) patients.

Methods: This study recruited 17 DMD patients, 32 DM1 patients, and 26 ALS patients. They underwent separate measurements of tongue and pharyngeal pressure under videofluoroscopy, swallowing $5 \mathrm{~mL}$ of barium water. We measured the largest change in pharyngeal pressure in the hypopharynx and the upper esophageal sphincter (UES) over several swallows.

Results: The mean tongue pressure (TP) was greatest in the DMD group than in the other groups $(p<0.01)$. There was a significant difference in pressure changes in the hypopharynx and UES between the DM1 group and other groups $(p<0.01)$. Significant correlations were observed between pressure change in the UES and the patient's age in the DMD group $(R=-0.500, p=0.045)$ and between pressure change in the hypopharynx and TP in the DM1 group $(R=0.421, p=0.016)$. There was a significant correlation between pressure change in the hypopharynx and disease severity in the ALS group $(R=0.435, p=0.030)$.

Conclusion: Patients with DMD, DM1, and ALS have weakness in the muscles involved in swallowing; however, the results of this study suggested that each disorder has a distinctive profile of impairment in the swallowing function.

Keywords: videomanofluorometry, tongue pressure, pharyngeal pressure, dysphagia, neuromuscular disease

\section{Introduction}

Patients with neuromuscular diseases (NMDs) often experience problems with swallowing during the course of the illness. Duchenne muscular dystrophy (DMD), myotonic dystrophy type 1 (DM1), and amyotrophic lateral sclerosis (ALS) are the most common diseases of NMDs which induce dysphagia due to muscle weakness. Previous studies utilizing videofluoroscopy (VF) images have demonstrated the characteristic findings. Patients with DMD often have reduced hyolaryngeal movement and poor pharyngeal clearance, which may be due to oropharyngeal weakness. ${ }^{1}$ Patients with DM1 generally have pharyngeal residue, and it often takes a prolonged period of time to clear this residue from the pharynx, ${ }^{2}$ whereas patients with ALS less often show signs of dysphagia on VF during the early stage of the condition, despite a progressively worsening of oropharyngeal and hypopharyngeal swallowing pressures. ${ }^{3}$ 
We previously reported the association between tongue pressure (TP) and VF findings of patients with DMD and DM1. ${ }^{4}$ Despite greater hyoid bone excursion, patients with DM1 showed more pharyngeal residue than the patients with DMD. However, the mechanisms underlying impaired pharyngeal clearance remain to be completely understood. The mechanism by which muscle weakness influences swallowing function is possibly different depending on the muscular diseases. The aim of this study was to clarify how pressure involved in swallowing results in difficulty of the passage of bolus from the pharynx to the esophagus in patients with NMDs. We analyzed the relationship between TP and videomanofluorometry (VMF) findings in patients with DMD, DM1, and ALS.

\section{Methods}

\section{Subjects}

This study enrolled 17 patients with DMD (all men; mean age 22.0 years; range $14-36$ years), 32 patients with DM1 (16 men and 16 women; mean age 47.8 years; range $15-68$ years), and 26 patients with ALS (10 men and 16 women; mean age 68.9 years; range 54-81 years) in the Department of Neurology at the Fukuoka University Hospital in Fukuoka City, Japan, and the Department of Neurology at the Neuromuscular Center of the National Omuta Hospital in Omuta City, Japan (Table 1). All diagnoses of DMD and DM1 were genetically confirmed after clinical diagnosis. All patients with DM1 were not congenital, but acquired. All patients with ALS were clinically and neurophysiologically assessed by board-certified neurologists of the Japanese Society of Neurology, and were diagnosed as probable or definite ALS according to the Awaji criteria. ${ }^{5}$ The ALS group was divided into two subgroups including a bulbar-onset $(n=13)$ and a limb-onset group ( $\mathrm{n}=13)$. The disease severity of ALS was assessed using the revised ALS Functional Rating Scale (ALSFRS-R). ${ }^{6}$ Furthermore, each patient was followed clinically for more than 1 year. Patients with other neurologic disorders were excluded. One DMD patient, three DM1 patients, and two ALS patients had tracheostomy tubes. This study was approved by the ethics committee of the Fukuoka University Hospital. Written informed consent was obtained from all participants.

\section{Diet-type score}

The ability to eat by mouth was assessed by scoring the following four feeding conditions on a scale of $0-4$ points: 4 points, total oral diet with no restrictions; 3 points, total oral intake with no special preparation, but must avoid specific
Table I Videofluoroscopic dysphagia scale

\begin{tabular}{|c|c|c|c|}
\hline \multirow{2}{*}{$\begin{array}{l}\text { Parameter } \\
\text { Oral phase score }\end{array}$} & \multicolumn{2}{|c|}{ Coded value } & \multirow[t]{2}{*}{ Score } \\
\hline & & & \\
\hline \multirow[t]{3}{*}{ Lip closure } & Intact & 0 & 4 \\
\hline & Inadequate & 2 & \\
\hline & None & 4 & \\
\hline \multirow[t]{3}{*}{ Bolus formation } & Intact & 0 & 6 \\
\hline & Inadequate & 3 & \\
\hline & None & 6 & \\
\hline \multirow[t]{3}{*}{ Mastication } & Intact & 0 & 8 \\
\hline & Inadequate & 4 & \\
\hline & None & 8 & \\
\hline \multirow[t]{4}{*}{ Apraxia } & None & 0 & 4.5 \\
\hline & Mild & 1.5 & \\
\hline & Moderate & 3 & \\
\hline & Severe & 4.5 & \\
\hline \multirow[t]{3}{*}{ Tongue-to-palate contact } & Intact & 0 & 10 \\
\hline & Inadequate & 5 & \\
\hline & None & 10 & \\
\hline \multirow[t]{4}{*}{ Premature bolus loss } & None & 0 & 4.5 \\
\hline & $<10 \%$ & 1.5 & \\
\hline & $10 \%-50 \%$ & 3 & \\
\hline & $>50 \%$ & 4.5 & \\
\hline \multirow[t]{2}{*}{ Oral transit time } & $<1.5$ & 0 & 3 \\
\hline & $>1.5$ & 3 & \\
\hline \multicolumn{4}{|l|}{ Pharyngeal phase score } \\
\hline Triggering of pharyngeal & Normal & 0 & 4.5 \\
\hline swallow & Delayed & 4.5 & \\
\hline \multirow[t]{4}{*}{ Vallecular residue } & None & 0 & 6 \\
\hline & $<10 \%$ & 2 & \\
\hline & $10 \%-50 \%$ & 4 & \\
\hline & $>50 \%$ & 6 & \\
\hline \multirow[t]{2}{*}{ Laryngeal elevation } & Normal & 0 & 9 \\
\hline & Impaired & 9 & \\
\hline \multirow[t]{4}{*}{ Pyriform sinus residue } & None & 0 & 13.5 \\
\hline & $<10 \%$ & 4.5 & \\
\hline & $10 \%-50 \%$ & 9 & \\
\hline & $>50 \%$ & 13.5 & \\
\hline Coating of pharyngeal & No & 0 & 9 \\
\hline wall & Yes & 9 & \\
\hline \multirow[t]{2}{*}{ Pharyngeal transit time } & $<1.0 \mathrm{~s}$ & 0 & 6 \\
\hline & $>1.0 \mathrm{~s}$ & 6 & \\
\hline \multirow[t]{5}{*}{ Aspiration } & None & 0 & 12 \\
\hline & Supraglottic & 6 & \\
\hline & penetration & & \\
\hline & Subglottic & 12 & \\
\hline & aspiration & & \\
\hline Total & & & 100 \\
\hline
\end{tabular}

foods or liquid items; 2 points, total oral intake of multiple consistencies requiring special preparation; 1 point, total oral intake of a single consistency; and 0 points, tube dependent. The diet types administered were recorded on each patient's medical chart. This method of assessing the diet type was based on the seven-level classification system used by the functional oral intake scale. ${ }^{7}$ The attending physician selected 
the most appropriate diet type and eating position after evaluating each patient's swallowing abilities based on VF findings.

\section{Measurement of TP}

TP was measured using a handy probe that consisted of a small balloon that was pressurized with air to a pressure of 19.6 kPa (JM-TPM; JMS, Hiroshima, Japan). ${ }^{8}$ Participants were required to compress the balloon onto the palate with their tongue for $\sim 7 \mathrm{~s}$, while applying maximum effort. The resulting increase in the inner pressure of the balloon was measured and recorded as TP. These tests were conducted thrice in a row, and the mean values were obtained. The previous study confirmed the reliability of intraindividual measurement in healthy individuals with the coefficient of variation, $5.64 \%{ }^{8}$

\section{VMF examination}

Swallowing function was evaluated using a modified bariumswallowing procedure that incorporated VMF imaging. ${ }^{9} \mathrm{VMF}$ images were acquired using a radiologic instrument (DR2000; Hitachi Medical Corp., Tokyo, Japan) and recorded via lateral projection onto a DVD recorder (DIGA; Panasonic, Osaka, Japan) running at 30 frames/s. Each participant voluntarily swallowed a $5 \mathrm{~mL}$ mixture of barium and water in an upright or reclining position due to scoliosis which made sitting difficult and uncomfortable. All recordings were analyzed frame-by-frame and scored on the basis of the VF dysphagia scale with a sum of 100 (Table 1). ${ }^{10}$

In the manometry, a four-site transducer probe using UniTip catheters (UNISENSOR AG, Attikon, Switzerland), ${ }^{11}$ with four circumferential sensors $2 \mathrm{~cm}$ apart, was passed transnasally. The sensors of channels 1-3 were positioned from the oropharynx to the hypopharynx, while the sensor of channel 4 was positioned at the upper esophageal sphincter (UES; Figure 1). Manometric data were superimposed on fluorographic images using a personal computer system and analyzed as intrabolus pressure. The convex waveforms of channels 1-3 and the concave waveform of channel 4 appeared simultaneously during a dry swallow. We measured the highest pressure peak value in the hypopharynx at the mean of channels 1-3 and the largest UES pressure change which was taken in channel 4 over the several repeated swallows (Figure 2).

\section{Data analysis}

The Steel-Dwass tests were used to compare group-wise differences in diet-type score and VF score, and the Bonferroni (Dunn) $t$-tests were done in swallowing pressures between

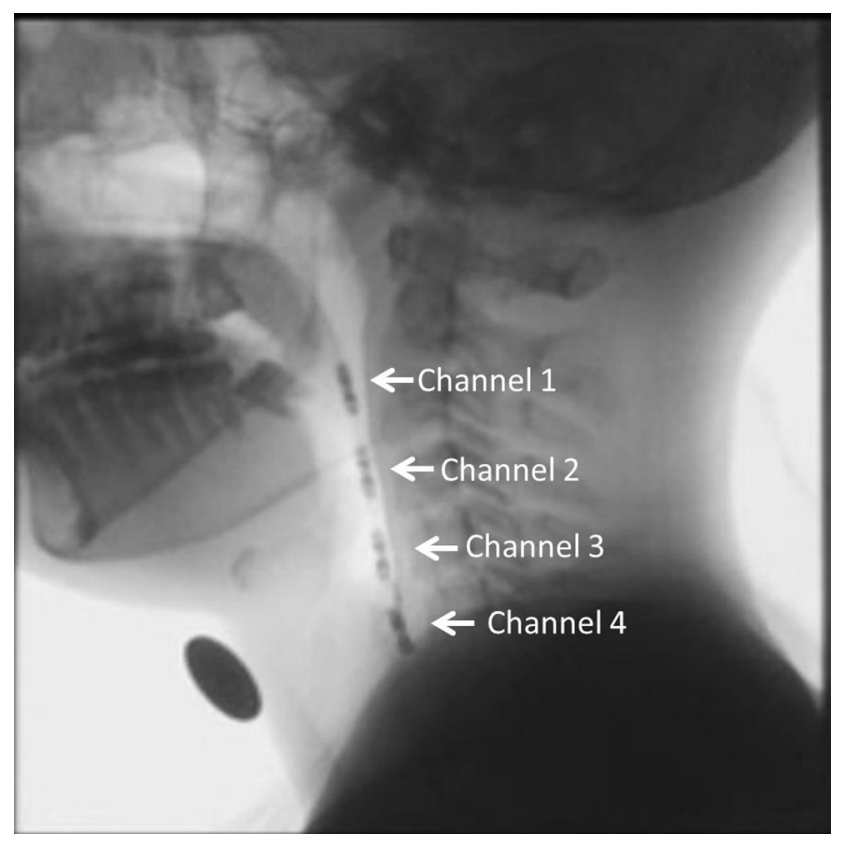

Figure I Position of the four sensors of the transducer probe in videomanofluorometry.

Notes: The sensors of channels I-3 were positioned from the oropharynx to the hypopharynx, while the sensor of channel 4 was positioned at the upper esophageal sphincter.

the patient groups. We compared all pairs to determine which medians were significantly different.

The Pearson correlation coefficient was applied to measure the linear relationships between swallowing pressures. Spearman's rank-order correlation was applied to measure the linear relationships between pressure measurements, age, VF score, and ALSFRS-R score. All statistical analyses were performed using SPSS 13.0 J for Windows (SPSS, Inc., Chicago, IL, USA). A $p$ value of $<0.05$ was considered statistically significant.

\section{Results}

The diet-type scores were not significantly different among the three groups (Table 2). The mean diet-type scores of the three groups were DMD 2.24, DM1 2.12, and ALS 1.92. The most frequent score in DMD and DM1 groups was point 3: soft rice and side dishes, and in DMD and ALS groups point 1: pureed diet. Regarding the number of diet type point 4: normal diet, 2 of 16 in DMD group, 4 of 32 in DM1 group, and 3 of 26 in ALS group were respectively counted, but it contained patients who rejected to accept the recommendation to adjust the diet type. As to the number of diet type point 1: pureed diet, 6 of 16 in DMD group, 2 of 32 in DM1 group, and 10 of 26 in ALS group were respectively observed. The VF score in the ALS group was significantly higher than those in the other two groups $(p<0.05)$. Focusing 


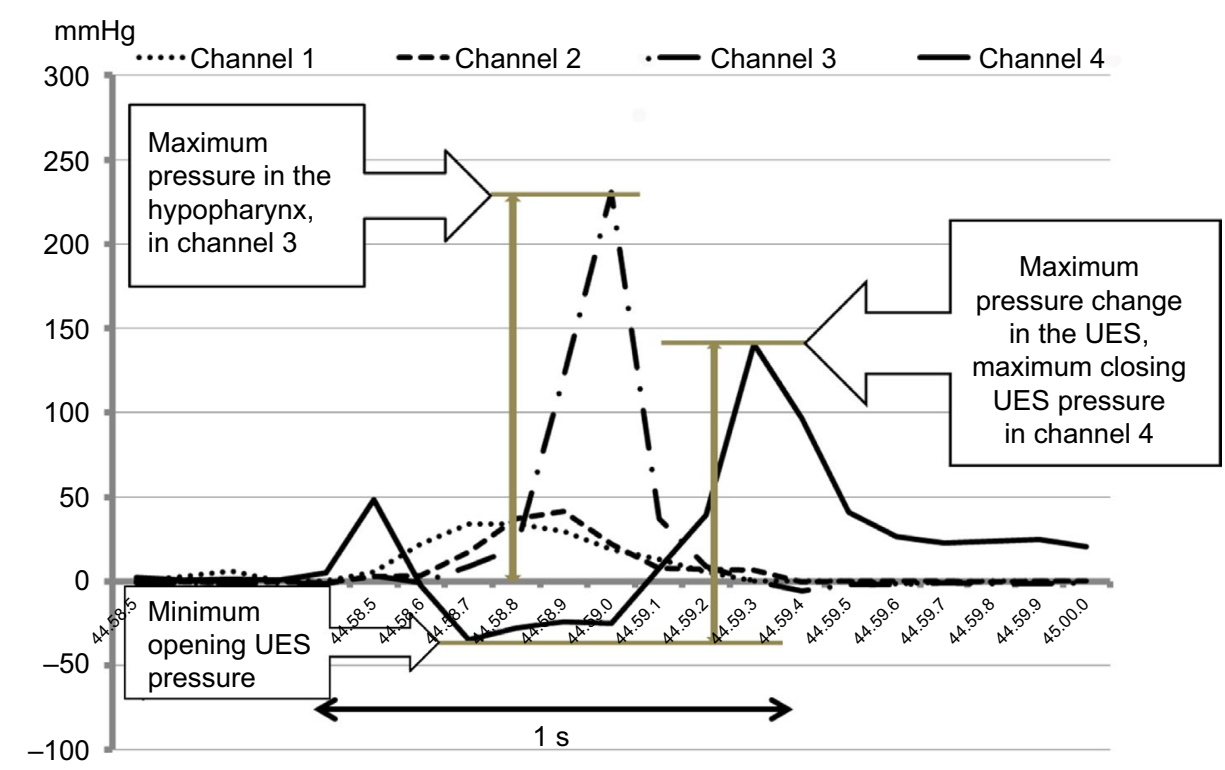

Figure 2 Maximum pressures in the hypopharynx in channels I-3 were measured from the highest peak of a waveform to the base line, and pressure change in the UES in channel 4 was measured from a higher peak, that is, maximum closing UES pressure, to the bottom of a waveform, that is, minimum opening UES pressure.

Abbreviation: UES, upper esophageal sphincter.

Table 2 Comparison of the three groups with regard to patient characteristics and swallowing pressures

\begin{tabular}{|c|c|c|c|c|}
\hline & DMD group & DMI group & ALS group & Reference value"I \\
\hline Age (years) & $22.0 \pm 5.5$ & $47.8 \pm 12.0$ & $68.9 \pm 7.1$ & \\
\hline Men:women & 17 men & $16: 16$ & $10: 16$ & \\
\hline Bulbar onset:limb onset & & & $13: 13$ & \\
\hline $\begin{array}{l}\text { Diet-type score (score, number of } \\
\text { patients) }\end{array}$ & $\begin{array}{l}2.24(0,0 ; 1,6 ; 2,3 ; 3 \\
6 ; 4,2)\end{array}$ & $\begin{array}{l}2.12(0,7 ; 1,2 ; 2,7 ; 3 \\
12 ; 4,4)\end{array}$ & $\begin{array}{l}1.92(0,3 ; 1,10 ; 2,2 ; 3 \\
8 ; 4,3)\end{array}$ & \\
\hline $\begin{array}{l}\text { Videofluoroscopy score (minimum- } \\
\text { maximum) }\end{array}$ & $7.5(2-16.5)$ & $12.2(2-27.5)$ & $20.6(0-59.5)^{* *}$ & \\
\hline Tongue pressure $(\mathrm{kPa})$ & $21.7 \pm 7.8 * *$ & $13.9 \pm 6.4$ & $13.5 \pm 10.2$ & \\
\hline $\begin{array}{l}\text { Data in healthy individuals (age } \\
\text { boundaries) }\end{array}$ & $41.7 \pm 9.7(20 \mathrm{~s})$ & $40.4 \pm 9.8(40 \mathrm{~s})$ & $37.6 \pm 8.8(60 \mathrm{~s})$ & \\
\hline \multicolumn{4}{|c|}{ Change in hypopharynx pressure $(\mathrm{mmHg})$} & (healthy volunteers) \\
\hline Channel I & $80.3 \pm 64.0$ & $48.2 \pm 51.6$ & $71.6 \pm 34.0$ & $71.1(50.2-142.3)$ \\
\hline Channel 2 & $78.3 \pm 118.1$ & $28.7 \pm 29.6$ & $82.9 \pm 43.1$ & $53.5(20-89.7)$ \\
\hline Channel 3 & $126.7 \pm 74.6$ & $76.3 \pm 73.9$ & $178.5 \pm 79.5$ & $62.5(47.3-14.8)$ \\
\hline Mean of channels I-3 & $95.1 \pm 42.4$ & $51.1 \pm 29.9 * *$ & $111.0 \pm 32.4$ & \\
\hline \multicolumn{5}{|l|}{ Change in UES pressure $(\mathrm{mmHg})$} \\
\hline Channel 4 & $217.9 \pm 135.4$ & $95.6 \pm 92.2 * *$ & $160.8 \pm 75.8$ & I 87.4 (70.1-287.2) \\
\hline
\end{tabular}

Notes: ${ }^{* *} p<0.01$. Differences in diet-type score and videofluoroscopy score were analyzed using the Steel-Dwass tests. Differences in swallowing pressures were analyzed using the Bonferroni (Dunn) $t$-tests.

Abbreviations: ALS, amyotrophic lateral sclerosis; DMI, myotonic dystrophy type I; DMD, Duchenne muscular dystrophy; UES, upper esophageal sphincter.

on the frequent subscores in VF score, poor bolus formation and aspiration more likely appeared in ALS group than in the other groups (ALS: $2.7 \pm 2.2$ and $2.1 \pm 3.7$, DMD: $1.2 \pm 1.5$ and 0 , and DM1: $1.2 \pm 1.7$ and $0.19 \pm 1.1$, respectively) and pyriform sinus residue more likely appeared in DM1 group than in the other groups (DM1: $5.6 \pm 3.0$, ALS: $3.1 \pm 4.2$, and DMD: $4.0 \pm 4.5$; Figure 3). The mean value of the TP in the DMD group was significantly higher than those in the other two groups $(p<0.01)$. In each group, TP was lower than the data of healthy individuals of the same generation..$^{12}$ The pressure changes in the hypopharynx and the UES were both significantly lower in the DM1 group than in the other two groups $(p<0.01)$. The data of the DM1 group were also lower than those of healthy volunteers. ${ }^{13}$ Moreover, the TP value in the ALS bulbar-onset group was significantly lower than that in the limb-onset group ( $p<0.05$; Table 3 ). 
In the DMD group, significant negative correlations were observed between patient age and TP $(R=-0.498, p=0.046$; Table 4) and between age and change in UES pressure ( $R=-0.500, p=0.045$; Table 4); however, there was no significant correlation between TP and UES pressures. In the DM1 group, although there was no significant correlation between patient age and pressure values, a significant positive

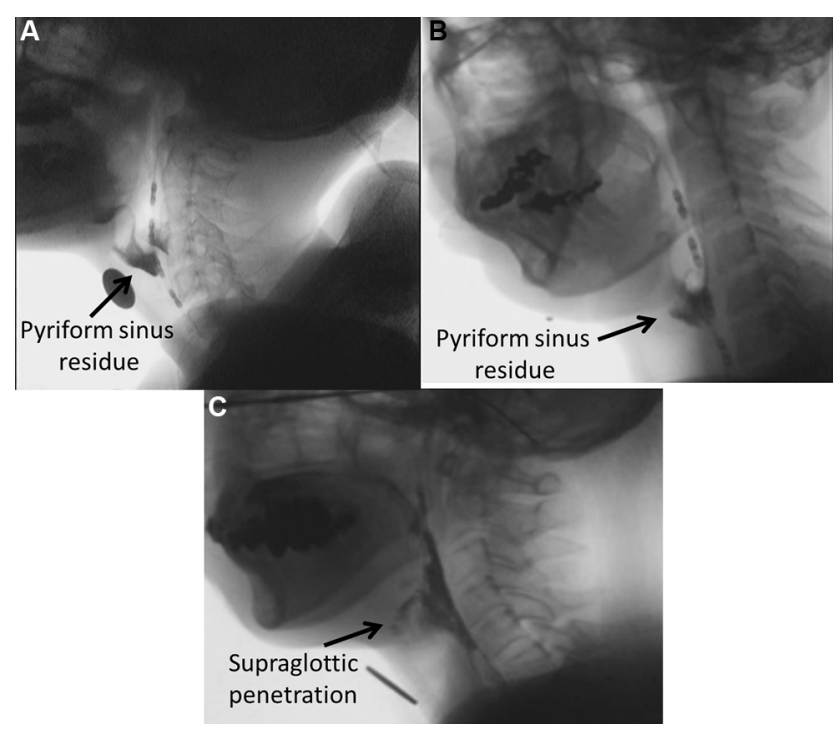

Figure 3 VF images of typical cases in each group; pyriform sinus residue in a patient with DMD and DMI ( $\mathbf{A}$ and $\mathbf{B}$, respectively) and supraglottic penetration in a patient with ALS (C).

Abbreviations: ALS, amyotrophic lateral sclerosis; DMI, myotonic dystrophy type I; DMD, Duchenne muscular dystrophy; VF, videofluoroscopy. correlation was observed between TP and hypopharynx pressure $(R=0.421, p=0.016$; Table 4$)$.

In contrast, the ALS group showed no significant correlations between patient age and pressure values, or between pressures at the various sites. However, a significant correlation was observed between ALSFRS-R score and the change in hypopharynx pressure $(R=0.435, p=0.030$; Table 5$)$. Focusing on the subscores in ALSFRS-R related to oral and swallowing function, speech, salivation, and swallowing, a significant correlation was found only between TP and salivation $(R=0.637, p=0.011)$. When the ALS group was stratified by the location of onset, the correlation between ALSFRS-R score and the pressure change in the hypopharynx was stronger in the limb-onset group $(R=0.593)$ than in the bulbar-onset group $(R=0.437)$.

All the groups did not show significant correlations between VF score and swallowing pressures.

\section{Discussion}

This is the first study to compare the swallowing pressure of patients with NMDs using VMF. Patients with DMD, DM1, and ALS have similar swallowing symptomology in the muscles involved in swallowing, but the results of this study suggested that each disease has distinctive characteristics.

The average TP in healthy individuals has been reported to be $41.7 \pm 9.7 \mathrm{kPa}$ for those in their $20 \mathrm{~s}, 40.4 \pm 9.8 \mathrm{kPa}$ for those in their $40 \mathrm{~s}$, and $37.6 \pm 8.8 \mathrm{kPa}$ for those in their $60 \mathrm{~s} .{ }^{12}$ The average TP values in acute stroke patients at a mean age

Table 3 Comparison between the bulbar-onset group and the limb-onset group in the ALS group

\begin{tabular}{llll}
\hline & ALS group & Bulbar-onset group & Limb-onset group \\
\hline Age (years) & $68.9 \pm 7.1$ & $71.2 \pm 6.6$ & $66.5 \pm 7.0$ \\
Men:women & $10: 16$ & $5: 8$ & $5: 8$ \\
Disease duration (years) & $2.7 \pm 3.8$ & $1.4 \pm 1.3$ & $3.9 \pm 5.1$ \\
Body mass index $\left(\mathrm{kg} / \mathrm{m}^{2}\right)$ & $18.4 \pm 3.4$ & $19.3 \pm 3.8$ & $17.5 \pm 2.9$ \\
ALSFRS-R (minimum-maximum) & $27.8(13-46)$ & $29.7(13-46)$ & $25.9(14-42)$ \\
Diet type (score, number of patients) & $1.92(0,3 ; 1,10 ; 2$, & $1.54(0,1 ; 1,7 ; 2,2 ; 3$, & $2.31(0,2 ; 1,3 ; 2,0 ;$ \\
& $2 ; 3,8 ; 4,3)$ & $3 ; 4,0)$ & $3,5 ; 4,3)$ \\
Videofluoroscopy score (minimum- & $20.6(0-59.5)$ & $25.0(0-59.5)$ & $16.2(2-38.5)$ \\
maximum) & & & $17.46 \pm 9.0 *$ \\
Tongue pressure (kPa) & $13.5 \pm 10.2$ & & $17.6 \pm 8.3$ \\
Change in hypopharynx pressure $(\mathrm{mmHg})$ & & $61.7 \pm 30.2$ & $81.5 \pm 35.8$ \\
Channel I & $71.6 \pm 34.0$ & $92.7 \pm 46.9$ & $73.1 \pm 38.3$ \\
Channel 2 & $82.9 \pm 43.1$ & $155.0 \pm 65.0$ & $202.1 \pm 88.0$ \\
Channel 3 & $178.5 \pm 79.5$ & $103.1 \pm 34.1$ & $118.9 \pm 29.7$ \\
Mean of channels I-3 & $111.0 \pm 32.4$ & & $180.5 \pm 71.0$ \\
Change in UES pressure $(\mathrm{mmHg})$ & & $141.1 \pm 78.0$ & \\
Channel 4 & $160.8 \pm 75.8$ & & \\
\hline
\end{tabular}

Notes: ${ }^{*} p<0.05$. Differences in ALSFRS-R, diet-type score and videofluoroscopy score were analyzed using the Steel-Dwass tests. Differences in swallowing pressures were analyzed using the Bonferroni (Dunn) $t$-tests.

Abbreviations: ALS, amyotrophic lateral sclerosis; ALSFRS-R, ALS functional rating scale; UES, upper esophageal sphincter. 
Table 4 Correlations between age and swallowing pressures and among swallowing pressures in the three groups of patients

\begin{tabular}{llll}
\hline & DMD group & DMI group & ALS group \\
\hline Age vs tongue pressure & $R=-0.498, p=0.046^{*}$ & $R=-0.108, p=0.547$ & $R=-0.104, p=0.635$ \\
Age vs hypopharynx pressure & $R=-0.014, p=0.955$ & $R=-0.019, p=0.914$ & $R=0.044, p=0.826$ \\
Age vs UES pressure & $R=-0.500, p=0.045^{*}$ & $R=-0.199, p=0.267$ & $R=-0.189, p=0.346$ \\
Tongue pressure vs hypopharynx pressure & $R=0.105, p=0.688$ & $R=0.421, p=0.016^{*}$ & $R=-0.026, p=0.909$ \\
Tongue pressure vs UES pressure & $R=0.353, p=0.164$ & $R=0.329, p=0.066$ & $R=0.197, p=0.379$ \\
Hypopharynx pressure vs UES pressure & $R=-0.277, p=0.282$ & $R=-0.110, p=0.549$ & $R=0.030, p=0.883$ \\
\hline
\end{tabular}

Notes: $* p<0.05$. The relationships between pressures and age were measured using the Spearman's rank-order correlation. The relationships between swallowing pressures were measured using the Pearson correlation coefficient.

Abbreviations: ALS, amyotrophic lateral sclerosis; DMI, myotonic dystrophy type I; DMD, Duchenne muscular dystrophy; UES, upper esophageal sphincter.

Table 5 Correlations between ALSFRS-R and swallowing pressures and among swallowing pressures in the ALS bulbar-onset and limb-onset groups

\begin{tabular}{llll}
\hline & ALS group & Bulbar-onset group & Limb-onset group \\
\hline ALSFRS- $R$ vs tongue pressure & $R=0.078, p=0.715$ & $R=-0.025, p=0.943$ & $R=0.084, p=0.767$ \\
ALSFRS- $R$ vs hypopharynx pressure & $R=0.435, p=0.030^{*}$ & $R=0.437, p=0.130$ & $R=0.593, p=0.040^{*}$ \\
ALSFRS-R vs UES pressure & $R=-0.034, p=0.864$ & $R=-0.088, p=0.76 \mathrm{I}$ & $R=0.177, p=0.540$ \\
BMI vs tongue pressure & $R=-0.148, p=0.509$ & $R=-0.087, p=0.823$ & $R=0.095, p=0.758$ \\
BMI vs hypopharynx pressure & $R=0.294, p=0.144$ & $R=0.535, p=0.060$ & $R=0.162, p=0.596$ \\
BMI vs UES pressure & $R=-0.049, p=0.811$ & $R=0.252, p=0.407$ & $R=-0.304, p=0.313$ \\
Tongue pressure vs hypopharynx pressure & $R=-0.026, p=0.909$ & $R=-0.310, p=0.417$ & $R=-0.060, p=0.845$ \\
Tongue pressure vs UES pressure & $R=0.197, p=0.379$ & $R=0.191, p=0.622$ & $R=-0.137, p=0.656$ \\
Hypopharynx pressure vs UES pressure & $R=0.030, p=0.883$ & $R=-0.285, p=0.344$ & $R=0.300, p=0.320$ \\
\hline
\end{tabular}

Notes: $*_{p}<0.05$. The relationships between pressures and ALSFRS-R were measured using the Spearman's rank-order correlation. The relationships between swallowing pressures were measured using the Pearson correlation coefficient.

Abbreviations: ALS, amyotrophic lateral sclerosis; ALSFRS-R, ALS functional rating scale; BMI, body mass index; UES, upper esophageal sphincter.

of 73.9 years and in patients with spinal and bulbar muscular atrophy at a mean age of 52.1 years have been reported to be $22.8 \pm 14.6$ and $15.3 \pm 6.4 \mathrm{kPa}$, respectively. ${ }^{13,14}$ The mean TP in our patients with DMD corresponded approximately to half of that of healthy individuals and was close to that of stroke patients, but was higher than that of patients with other NMDs. Although the patients with DMD had relatively high TP and UES pressures, these decreased with age; a low pressure in the hypopharynx, especially in channel 3 , was noticeable. The discrepancy in pressure changes between the hypopharynx and UES may result in difficulty in the passage of bolus. Furthermore, maintaining a high TP does not necessarily mean retaining the function of the tongue, since the enlarged tongue of a DMD patient often has a reduced ability to transport a bolus from the mouth to the pharynx..$^{15}$

Significantly reduced pressures were observed in the tongue and pharynx of patients with DM1. The mean TP in the DM1 group was close to that of patients with spinal and bulbar muscular atrophy. ${ }^{14}$ We have previously reported deterioration of the masticatory and lingual muscles in patients with DM1, ${ }^{4}$ suggesting that DM1 completely impairs oropharyngeal function. In fact, patients with DM1 in this study showed a significant correlation between TP and the pressure change in the hypopharynx. The long duration and slow progression of this condition, leading to reduced consciousness ${ }^{16}$ of oropharyngeal dysfunction, may be associated with the lack of a correlation between patient age and the pressures investigated in this study. However, the low pressures found here may induce a high frequency of food aspiration and suffocation. These results also suggest the possibility that in patients with DM1, weakness in the hypopharynx and UES muscles might be predicted by measuring TP.

In our study, patients with ALS showed significantly decreased TP compared with the DMD group, with particularly low pressures in the bulbar-onset group, but maintained a high pressure change in the hypopharynx relative to the other two groups. In the limb-onset group, the pressure change in the hypopharynx decreased with impairment in activities of daily living. Based on the results, the significant difference in TP and little difference in pharyngeal pressures between the two onset groups, dysphagia in patients with ALS at an early stage presumably begins to show a symptom of tongue muscle weakness, and TP measurement is, therefore, important in assessing swallowing function. 
Several studies have examined the pharyngeal pressure of patients with NMD using manometry. Modolell et al evaluated the pharyngoesophageal motility of 18 patients with DM1. ${ }^{17}$ The pharyngeal contraction amplitude and UES basal pressure of this group ( $24 \pm 2$ and $18 \pm 3 \mathrm{mmHg}$, respectively) were diminished to less than half of the values of the control group. Although the difference in the pressure values compared with our results would be due to the use of a different manometric procedure, the degrees of pressure reduction seen in both studies are assumable. Moreover, Modolell et al referred to an absence of myotonia in postcontraction relaxation, and we also failed to detect myotonia. These results suggest that the difficulty in the passage of food in patients with DM1 might be mainly caused not by myotonia, but by muscle weakness.

Three previous manometric studies have been conducted including patients with ALS. Higo et al investigated 21 patients with ALS using VMF, and their oropharyngeal swallowing pressures decreased to $\sim 50 \mathrm{mmHg}$ within 6 months of the appearance of bulbar symptoms. ${ }^{3}$ Furthermore, the study revealed a progressive decrease in pharyngeal contraction from the oropharynx to the hypopharynx. Most of the patients maintained normal UES relaxation, but some patients with UES spasm showed aspiration. Goeleven et al studied 40 patients with ALS and revealed low tonguedriving forces and pharyngeal contraction amplitudes, which were $\sim 70 \%$ of the normal data. ${ }^{18}$ They concluded that these reduced functions appeared to cause pharyngeal retention and aspiration. On the other hand, relaxation of the UES was at a normal level. Solazzo et al reported the contraction pressures of ten patients with ALS, which were $\sim 70 \mathrm{mmHg}$ in the pharynx and $161.2 \mathrm{mmHg}$ in the UES and were not different to those of healthy volunteers. ${ }^{11}$ They indicated an increase in the pharyngeal contraction time and in residual pressure after relaxation of the UES. Our data are similar to those of Solazzo et al; ${ }^{11}$ therefore, the contraction pressures of the pharynx and UES in our patients with ALS are also thought to be equivalent to those of healthy people. Weikamp et al reported that $37 \%$ of ALS patients, especially $67 \%$ of bulbar-onset patients, had decreased tongue strength, which was an independent prognostic factor for survival time in ALS patients. ${ }^{19}$ Considering the results of these previous studies and our study, tongue weakness, along with slightly reduced pharyngeal contraction, seems to be the main cause of swallowing disturbance in patients with ALS.

By the way, there were only a few patients who exhibited aspiration on VF image in the DM1 and ALS groups. Although many participants had low swallowing pressure and showed at least moderate pharyngeal residue, these findings do not necessarily lead to aspiration on VF. Aspiration is evoked not only by lowered swallowing pressure or pharyngeal residue but also by other various factors such as delayed swallowing reflex or respiration issues. It could be argued that assessing only swallowing pressures is not enough and image examinations of VF or videoendoscopy are essential to detect a symptom of aspiration.

\section{Limitations}

This study has some limitations. First, the hypopharynx and UES pressures were measured while the patients swallowed water, but TP was measured without the patients swallowing according to an instruction. Recently, a new TP measuring system with an ultrathin sensor sheet has enabled TP to be measured during swallowing. ${ }^{20}$ However, we placed priority on the convenience of measurement and analysis and chose to use a handy probe instead. TP was, therefore, not measured during swallowing, but this method could be treated as an indication of swallowing function. ${ }^{4}$ Second, we did not use a high-resolution manometry (HRM) system. HRM system has many sensors and gives us more information than the four-sensor system in this study does, but the many sensors and the thicker catheter in HRM system inflict suffering on patients. We considered that the information obtained by the four-sensor system was suitably qualified for comparing characteristics in swallowing pressures between NMDs and gave priority on decreasing the burden on patients during the examination. Third, we were unable to adjust for the confounding effects of age and disease progression, considering the sample heterogeneity in disease onset and progression. However, differences in disease onset or progression make it difficult to adjust for these possible confounding factors. Therefore, we tried to compensate for these differences by determining the diet-type score instead.

Despite these limitations, our results provide insight into the pathogenic mechanisms underlying dysphagia in patients with NMDs. In patients with DMD, measuring only the TP may not be sufficient to assess swallowing disturbances due to the complexity of weakness in the tongue, pharynx, and UES. Conversely, in patients with DM1, it is possible that swallowing dysfunction can be conveniently evaluated by measuring TP based on the integrally progressed muscle weakness. On the other hand, it is perhaps important to investigate tongue function in patients with ALS because of its influence on pharyngeal phase. The current data on pharynx and UES pressure changes in patients with NMD indicate the necessity of interpreting TP values differently 
depending on the specific NMD. Furthermore, TP is not only an important assessment for swallowing function in NMD patients but also a huge potential for the future rehabilitation to reduce pharyngeal residue, ${ }^{21}$ and further investigation will confirm these suggestions from this study.

\section{Conclusion}

Although most of the patients with DMD in this study had relatively high TP and UES pressure, these pressures tended to decrease with aging. Significantly decreased pressures in the tongue and pharynx were observed in patients with DM1. Patients with ALS showed significantly decreased TP, but maintained a high hypopharynx pressure; the latter, however, decreased with impairment in activities of daily living. Each of the three disorders has distinctive profile of impairment of swallowing function; therefore, it is essential that physicians provide the patients with distinct training for swallowing.

\section{Acknowledgment}

This work was supported by Japan Society for the Promotion of Science (JSPS) KAKENHI Grant number (25463276).

\section{Disclosure}

The authors report no conflicts of interest in this work.

\section{References}

1. Aloysius A, Born P, Kinali M, Davis T, Pane M, Mercuri E. Swallowing difficulties in Duchenne muscular dystrophy: indications for feeding assessment and outcome of videofluroscopic swallow studies. Eur J Paediatr Neurol. 2008;12(3):239-245.

2. Leonard RJ, Kendall KA, Johnson R, McKenzie S. Swallowing in myotonic muscular dystrophy: a videofluoroscopic study. Arch Phys Med Rehabil. 2001;82(7):979-985.

3. Higo R, Tayama N, Watanabe T, Nitou T. Videomanofluorometric study in amyotrophic lateral sclerosis. Laryngoscope. 2002;112(5):911-917.

4. Umemoto G, Furuya H, Kitashima A, Sakai M, Arahata H, Kikuta T. Dysphagia in Duchenne muscular dystrophy vs. myotonic dystrophy type 1. Muscle Nerve. 2012;46(4):490-495.

5. de Carvalho M, Dengler R, Eisen A, et al. Electrodiagnostic criteria for diagnosis of ALS. Clin Neurophysiol. 2008;119(3):497-503.
6. Cedarbaum JM, Stambler N, Malta E, et al. The ALSFRS-R: a revised ALS functional rating scale that incorporates assessments of respiratory function. BDNFALS Study Group (Phase III). J Neurol Sci. 1999; 169(1-2):13-21.

7. Crary MA, Mann GD, Groher ME. Initial psychometric assessment of a functional oral intake scale for dysphagia in stroke patients. Arch Phys Med Rehabil. 2005;86(8):1516-1520.

8. Hayashi R, Tsuga K, Hosokawa R, Yoshida M, Sato Y, Akagawa Y. A novel handy probe for tongue pressure measurement. Int J Prosthodont. 2002;15(4):385-388.

9. Logemann JA. Role of the modified barium swallow in management of patients with dysphagia. Otolaryngol Head Neck Surg. 1997;116(3): 335-338.

10. Han TR, Paik NJ, Park JW, Kwon BS. The prediction of persistent dysphagia beyond six months after stroke. Dysphagia. 2008;23(1):59-64.

11. Solazzo A, Monaco L, Vecchio LD, et al. Earliest videofluoromanometric pharyngeal signs of dysphagia in ALS patients. Dysphagia. 2014; 29(5):539-544.

12. Utanohara Y, Hayashi R, Yoshikawa M, Yoshida M, Tsuga K, Akagawa Y. Standard values of maximum tongue pressure taken using newly developed disposable tongue pressure measurement device. Dysphagia. 2008;23(3):286-290.

13. Nakamori M, Hosomi N, Ishikawa K, et al. Prediction of pneumonia in acute stroke patients using tongue pressure measurements. PLoS One. 2016;11(11):e0165837.

14. Mano T, Katsuno M, Banno H, et al. Tongue pressure as a novel biomarker of spinal and bulbar muscular atrophy. Neurology. 2014;82(3): 255-262.

15. Umemoto G, Furuya H, Arahata H, et al. Relationship between tongue thickness and tongue pressure in neuromuscular disorders. Neurology Clin Neurosci. 2016;4(4):142-145.

16. Baldanzi S, Bevilacqua F, Lorio R, et al. Disease awareness in myotonic dystrophy type 1: an observational cross-sectional study. Orphanet $J$ Rare Dis. 2016;11:34

17. Modolell I, Mearin F, Baudet JS, Gámez J, Cervera C, Malagelada JR. Pharyngo-esophageal motility disturbances in patients with myotonic dystrophy. Scand J Gastroenterol. 1999;34(9):878-882.

18. Goeleven A, Robberecht W, Sonies B, Carbonez A, Dejaeger E. Manofluorographic evaluation of swallowing in amyotrophic lateral sclerosis and its relationship with clinical evaluation of swallowing. Amyotroph Lateral Scler. 2006;7(4):235-740.

19. Weikamp JG, Schelhaas HJ, Hendriks JC, de Swart BJ, Geurts AC. Prognostic value of decreased tongue strength on survival time in patients with amyotrophic lateral sclerosis. J Neurol. 2012;259(11):2360-2365.

20. Hamanaka-Kondoh S, Kondoh J, Tamine K, et al. Tongue pressure during swallowing is decreased in patients with Duchenne muscular dystrophy. Neuromuscul Disord. 2014;24(6):474-481.

21. Steele CM, Bayley MT, Peladeau-Pigeon M, et al. A randomized trial comparing two tongue-pressure resistance training protocols for poststroke dysphagia. Dysphagia. 2016;31(3):452-461.
Degenerative Neurological and Neuromuscular Disease

\section{Publish your work in this journal}

Degenerative Neurological and Neuromuscular Disease is an international, peer-reviewed, open access journal focusing on research into degenerative neurological and neuromuscular disease, identification of therapeutic targets and the optimal use of preventative and integrated treatment interventions to achieve improved outcomes, enhanced 